TITLE:

\title{
Separator-type robust stability theorem of sampled-data systems allowing noncausal LPTV scaling
}

\author{
$\operatorname{AUTHOR}(\mathrm{S})$ : \\ Hagiwara, Tomomichi
}

\section{CITATION:}

Hagiwara, Tomomichi. Separator-type robust stability theorem of sampled-data systems allowing noncausal LPTV scaling. Automatica 2009, 45(8): 1868-1872

ISSUE DATE:

2009-08

URL:

http://hdl.handle.net/2433/84545

\section{RIGHT:}

c 2009 Elsevier Ltd. All rights reserved.; この論文は出版社版でありませ ん。引用の際には出版社版をご確認ご利用ください。; This is not the published version. Please cite only the published version. 


\title{
Separator-Type Robust Stability Theorem of Sampled-Data Systems Allowing Noncausal LPTV Scaling
}

\author{
Tomomichi Hagiwara ${ }^{a}$ \\ ${ }^{a}$ Department of Electrical Engineering, Kyoto University, Kyotodaigaku-Katsura, \\ Nishikyo-ku, Kyoto 615-8510, Japan.
}

\begin{abstract}
This paper derives a necessary and sufficient condition for robust stability of sampleddata systems, which is stated by using the notion of separators that are dealt with in an operator-theoretic framework. Such operator-theoretic treatment of separators provides a new perspective, which we call noncausal linear periodically time varying scaling and leads to reducing conservativeness in robust stability analysis. A numerical example is given to demonstrate the results.
\end{abstract}

Key words: Nyquist criterion; quadratic separator; fast-lifting; LPTV scaling.

\section{Introduction}

The robustness study of control systems with digital controllers has attracted great attention since the late 1980s, and it is widely recognized these days that such control systems should be treated as sampled-data systems [Chen and Francis (1995)]. In this paper, based on the lifting-based transfer operators [e.g., Yamamoto (1994)], we derive a new necessary and sufficient condition for robust stability of sampled-data systems, which is stated by using the notion of separators [Iwasaki and Hara (1998)]. It is well-known that various robust stability analysis methods in continuous/discrete-time can be interpreted from the viewpoint of separators, and the condition derived in this paper can partly be regarded as an extension to the sampled-data setting. It is thus expected to provide a solid basis for a new type of effective robust stability analysis for sampled-data systems. Indeed, due to the operator theoretic treatment of separators, the condition quite naturally leads to a novel approach called (causal/noncausal) linear periodically time-varying (LPTV) scaling [Hagiwara and Mori (2008), Hagiwara (2006), Hagiwara and Umeda (2007)], which is

Email address: hagiwara@kuee.kyoto-u.ac.jp (Tomomichi Hagiwara). 
very effective in reducing the conservativeness in the robust stability analysis of sampled-data systems.

The contents of this paper are as follows. In Section 3, we first derive a Nyquist stability criterion of sampled-data systems in terms of their transfer operators, which is with full generality compared with a similar study in Hagiwara (2002). Based on this criterion, we give some preliminary result that motivates the main subject of this paper. Then, in Section 4, we derive our main result, which is a general separator-type theorem about the necessary and sufficient condition for robust stability of sampled-data systems. Inspired by the structure of the class of separators, we introduce noncausal LPTV scaling in Section 5 and study a numerical example to demonstrate the significance of the arguments in this paper.

In the following, the Hilbert space $\left(L_{2}[0, h)\right)^{m}$ will be denoted by $\mathcal{K}_{m}$ for short.

\section{Lifting-Based Transfer Operators}

This paper is concerned with robust stability of the sampled-data system $\Sigma_{\Delta}$ shown in Fig. 1, where $P, \Psi$ and $\Delta$ denote the continuous-time generalized plant, the digital controller and the uncertainty, respectively, while $\mathcal{S}$ and $\mathcal{H}$ denote the ideal sampler and the zero-order hold, respectively. It is useful to consider the open-loop mapping from $w$ to $z$, which we call the open-loop sampled-data system for lack of better terminologies, and denote it by $\Sigma_{0}$. By introducing an appropriate matrix $\mathcal{A}$ and operators $\mathcal{B}, \mathcal{C}$ and $\mathcal{D}$, the transfer operator of $\Sigma_{0}$ is given by $\widehat{G}(\zeta):=\mathcal{C}(\zeta I-\mathcal{A})^{-1} \mathcal{B}+\mathcal{D}: \mathcal{K}_{m} \rightarrow \mathcal{K}_{p}$.

Let us denote by $D_{11}$ the direct feedthrough matrix of $P$ from $w$ to $z$ (as well as the associated multiplication operator). Then, the operator $\mathcal{D}_{0}:=\mathcal{D}-D_{11}$ is a Hilbert-Schmidt operator and thus is compact. Hence, $\widehat{G}(\zeta)$ is compact if and only if $D_{11}=0$. We can also introduce the transfer operator $\widehat{\Delta}(\zeta)$ of $\Delta$.

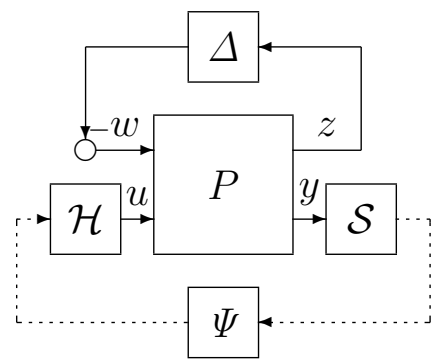

Fig. 1. Uncertain closed-loop sampled-data system $\Sigma_{\Delta}$. 


\section{Fundamental Stability Criteria for Sampled-Data Systems}

In this section, we first consider the stability condition for the case $\Delta=I$. For simplicity, we denote the corresponding sampled-data system by $\Sigma_{c}$ and call it the closed-loop sampled-data system. A general Nyquist stability criterion for $\Sigma_{c}$ is developed in Subsection 3.1, and robust stability of $\Sigma_{\Delta}$ with general $\Delta$ is studied in Subsection 3.2. The arguments will play a crucial role in the following arguments.

\subsection{Generalized Nyquist Stability Criterion of Sampled-Data Systems}

A Nyquist stability criterion of sampled-data systems was derived in Hagiwara (2002) under some restrictive assumptions that ensure $\widehat{G}(\zeta)$ to be a trace class operator; the use of the standard operator determinant naturally led to such assumptions, and the key that enables us to remove such assumptions is the use of the 2-regularized determinant (Böttcher and Silbermann (1990), Gohberg et al. (2000)), which was first introduced in Zhou and Hagiwara (2005) to the control field. We begin with the following assumption:

A0 The matrix $D_{11}$ has no eigenvalue at -1 .

This assumption is necessary and sufficient for $\Sigma_{c}$ to be well-posed, and also ensures $I+\mathcal{D}$ to be invertible. Also, it is easy to see that the closed-loop sampled-data system $\Sigma_{c}$ is internally stable if and only if $\mathcal{A}_{c}:=\mathcal{A}-\mathcal{B}(I+$ $\mathcal{D})^{-1} \mathcal{C}$ is a stable matrix in the discrete-time sense. To study the stability condition for $\mathcal{A}_{c}$, we define $\mathcal{A}^{\prime}=\mathcal{A}, \mathcal{B}^{\prime}=\mathcal{B}, \mathcal{C}^{\prime}=\left(I+D_{11}\right)^{-1} \mathcal{C}, \mathcal{D}^{\prime}=$ $\left(I+D_{11}\right)^{-1} \mathcal{D}_{0}$ and the corresponding modified transfer operator $\widehat{G}^{\prime}(\zeta)$. In fact, we are applying the well-known loop-shifting technique here, and this modified transfer operator is nothing but that of the modified open-loop sampled-data system $\Sigma_{0}^{\prime}$ obtained by replacing the generalized plant $P$ in $\Sigma_{0}$ by $P^{\prime}:=$ $\operatorname{diag}\left[\left(I+D_{11}\right)^{-1}, I\right]\left(P-\operatorname{diag}\left[D_{11}, 0\right]\right)$. We have $\mathcal{A}_{c}^{\prime}:=\mathcal{A}^{\prime}-\mathcal{B}^{\prime}\left(I+\mathcal{D}^{\prime}\right)^{-1} \mathcal{C}^{\prime}=\mathcal{A}_{c}$, and thus the stability analysis of $\Sigma_{c}$ can be restated as that of the closed-loop system $\Sigma_{c}^{\prime}$ corresponding to the modified open-loop sampled-data system $\Sigma_{0}^{\prime}$, where the advantage of studying the latter problem instead of the former lies in that the " $D_{11}$ matrix" has disappeared in the associated generalized plant $P^{\prime}$. It then follows that the operator $\mathcal{D}^{\prime}$ is a Hilbert-Schmidt operator, and thus so is the modified transfer operator $\widehat{G}^{\prime}(\zeta)$. Hence we can consider the 2-regularized determinant of $I+\widehat{G}^{\prime}(\zeta)$, about which we can derive

$$
\operatorname{det}_{2}\left(I+\widehat{G}^{\prime}(\zeta)\right) \cdot \exp (\eta(\zeta))=\frac{\operatorname{det}\left(\zeta I-\mathcal{A}_{c}\right)}{\operatorname{det}(\zeta I-\mathcal{A})}, \eta(\zeta)=\operatorname{tr}\left(\mathcal{B C}^{\prime}(\zeta I-\mathcal{A})^{-1}\right)(1)
$$

by following a similar procedure in the continuous-time setting, applying the properties of the 2-regularized determinant, and using the fact that $\mathcal{D}^{\prime}$ is a Volterra operator so that it has no (nonzero) eigenvalues (see Hagiwara and Tsuruguchi (2004) for details). Now, we introduce the following assumptions. 
A1 The matrix $\mathcal{A}$ has no eigenvalue on $\partial \mathbf{D}$ ( $\mathbf{D}$ denotes the open unit disk).

A2 $\operatorname{det}_{2}\left(I+\widehat{G}^{\prime}(\zeta)\right) \neq 0 \quad(\forall \zeta \in \partial \mathbf{D})$

We then have the following generalized Nyquist stability criterion.

Theorem 1 Under $\mathbf{A} \mathbf{0}, \mathbf{A} \mathbf{1}$ and $\mathbf{A} \mathbf{2}$, let $\mu_{0}^{+}$denote the number (algebraic multiplicities taken into account) of the eigenvalues of the matrix $\mathcal{A}$ in $\mathbf{D}$, and let $\nu$ denote the number of the anticlockwise encirclements of the Nyquist locus of $\operatorname{det}_{2}\left(I+\widehat{G}^{\prime}(\zeta)\right) \cdot \exp (\eta(\zeta))$ around the origin. Then, the closed-loop sampleddata system $\Sigma_{c}$ is well-posed and internally stable if and only if $\nu=\mu_{0}^{+}$.

Proof. The well-posedness assertion follows from the assumption A0. Thus, it is enough to prove that under the assumptions A0-A2, $\Sigma_{c}$ is internally stable if and only if $\nu=\mu_{0}^{+}$. It follows readily from $(1)$ that $\operatorname{det}_{2}\left(I+\widehat{G}^{\prime}(\zeta)\right)$. $\exp (\eta(\zeta))$ is meromorphic in $\zeta$, and by $\mathbf{A} \mathbf{1}$ and $\mathbf{A} \mathbf{2}$, the matrix $\mathcal{A}_{c}$ has $\mu_{0}^{+}-\nu$ eigenvalues on $\mathbf{D}$ by the argument principle. Since $\Sigma_{c}$ is internally stable if and only if $\mathcal{A}_{c}$ has no eigenvalues on $\mathbf{D}$, the stability condition follows. Q.E.D.

Remark 2 A0 and A2, which are necessary for well-posedness and stability of $\Sigma_{c}$, can be combined into the condition that $\sigma(\widehat{G}(\zeta)) \not \supset-1(\forall \zeta \in \partial \mathbf{D})$.

\subsection{Basic Robust Stability Theorem of Separator-Type}

In this section, we state a necessary and sufficient robust stability theorem for $\Sigma_{\Delta}$; it uses the notion of separators [Iwasaki and Hara (1998)] and thus we call it a separator-type robust stability theorem. It shares the important features of the robust stability analysis with separators [Iwasaki and Hara (1998)], and will be quite useful for unified treatment of various robust stability analysis and also in reducing the conservativeness in the robust stability analysis.

In this paper, $\Delta$ is assumed to be finite-dimensional and LTI for simplicity, and let us introduce

$$
P_{\Delta}:=P \operatorname{diag}[\Delta, I]
$$

Then, $\Sigma_{\Delta}$ can be identified with $\Sigma_{c}$ with $P$ replaced by $P_{\Delta}$, and thus the Nyquist stability criterion applies to $\Sigma_{\Delta}$. This idea together with some continuity arguments turns out to lead to a separator-type robust stability theorem. We begin with a definition that is necessary to state such a theorem.

Definition 3 The set of measurable, essentially bounded, Hermitian matrix functions $\Phi:[0, h) \rightarrow \mathbf{C}^{m \times m}$ is denoted by $\boldsymbol{\Phi}^{m \times m}$. The set of operators of multiplication by $\Phi \in \Phi^{m \times m}$, i.e., $\Lambda: \mathcal{K}_{m} \rightarrow \mathcal{K}_{m}, f(\cdot) \mapsto \Phi(\cdot) f(\cdot)$, is denoted by $\boldsymbol{\Lambda}^{m \times m}$. The set of operators $\Theta=\Lambda+K$ with $\Lambda \in \boldsymbol{\Lambda}^{m \times m}$ and a linear selfadjoint compact operator $K$ on $\mathcal{K}_{m}$ is denoted by $\boldsymbol{\Theta}_{0}^{m \times m}$. When $m$ is clear, we 
simply denote these sets by $\boldsymbol{\Phi}, \boldsymbol{\Lambda}$ and $\boldsymbol{\Theta}_{0}$, respectively.

We can now derive the following separator-type of theorem, whose proof will be deferred to Section 4 .

Theorem 4 Suppose that the open-loop sampled-data system $\Sigma_{0}$ is internally stable, and that $\boldsymbol{\Delta}$ is a set such that (i) every $\Delta \in \boldsymbol{\Delta}$ is FDLTI and internally stable, and (ii) $\kappa \Delta \in \boldsymbol{\Delta}$ whenever $\Delta \in \boldsymbol{\Delta}$ and $0<\kappa \leq 1$. Then, $\Sigma_{\Delta}$ is wellposed and internally stable for every $\Delta \in \boldsymbol{\Delta}$ if and only if there exists $\Theta \in \boldsymbol{\Theta}_{0}$ possibly dependent on $\zeta \in \partial \mathbf{D}$ and $\varepsilon>0$ possibly dependent on $\Delta$ such that

$$
\begin{aligned}
& {\left[\begin{array}{ll}
I & \widehat{G}(\zeta)^{*}
\end{array}\right] \Theta\left[\begin{array}{c}
I \\
\widehat{G}(\zeta)
\end{array}\right] \leq 0, \quad \forall \zeta \in \partial \mathbf{D}} \\
& {\left[-\widehat{\Delta}(\zeta)^{*} I\right] \Theta\left[\begin{array}{c}
-\widehat{\Delta}(\zeta) \\
I
\end{array}\right] \geq \varepsilon I, \quad \forall \Delta \in \boldsymbol{\Delta}, \forall \zeta \in \partial \mathbf{D}}
\end{aligned}
$$

The operator $\Theta$ is called a separator in the above theorem. This theorem inherits the features of the corresponding results in the continuous-time and discrete-time cases [Iwasaki and Hara (1998)] that have clarified the importance of separators. However, it does not always provide a systematic way to find an appropriate $\Theta \in \boldsymbol{\Theta}_{0}$ satisfying (3) and (4), as is the case with the continuous-time/discrete-time setting. Hence, what we could do with Theorem 4 is usually to use it only as a sufficient condition; we only consider some tractable subset of $\boldsymbol{\Theta}_{0}$ and check if there exists some $\Theta$, within such a subset, satisfying these two conditions. With this in mind, there is still a chance to generalize Theorem 4 further. That is, even though Theorem 4 already gives a necessary and sufficient condition, we could give a generalized statement of the necessary and sufficient condition which - when viewed as giving only a sufficient condition by restricting only to some tractable subset of all possible separators - leads to a less conservative sufficient condition. This corresponds to further extending the class of separators, and the remaining part of this paper is devoted to such discussions. Section 4 gives the extended class of separators, while Section 5 demonstrates the significance of such an extension by introducing what we call noncausal linear periodically time-varying (LPTV) scaling.

\section{Fast-Lifting and A Generalized Robust Stability Theorem of Separator-Type}

Inspired by the class $\boldsymbol{\Lambda}$ introduced in Subsection 3.2, a novel technique called (causal) linear periodically time-varying (LPTV) scaling for sampled-data systems has been discussed in our recent study [Hagiwara and Mori (2008)]. The purpose of this section is to extend Theorem 4 by introducing a set $\boldsymbol{\Gamma}(\supset \boldsymbol{\Lambda})$ such that the statement of this theorem remains true under the replacement of 
$\boldsymbol{\Lambda}$ with $\boldsymbol{\Gamma}$. The newly introduced class $\boldsymbol{\Gamma}$, together with another relevant class $\widetilde{\boldsymbol{\Gamma}}$, plays a crucial role for allowing us to introduce noncausal LPTV scaling.

\subsection{Fast-Lifting and the Classes $\boldsymbol{\Gamma}$ and $\widetilde{\boldsymbol{\Gamma}}$}

In this subsection, we introduce a new class $\boldsymbol{\Gamma}(\supset \boldsymbol{\Lambda})$ and the accordingly extended class $\Theta\left(\supset \boldsymbol{\Theta}_{0}\right)$ of separators, which play a crucial role in the generalization of Theorem 4 . We first need some preliminary arguments for introducing another relevant class $\widetilde{\boldsymbol{\Gamma}}$ together with what we call fast-lifting.

We begin with the introduction of fast-lifting. Suppose $x \in \mathcal{K}_{m}$. Take a positive integer $N$, define $h^{\prime}=h / N$ and $\mathcal{K}_{m}^{\prime}=\left(L_{2}\left[0, h^{\prime}\right)\right)^{m}$, and then define $x^{(l)} \in$ $\mathcal{K}_{m}^{\prime}(l=1, \cdots, N)$ by $x^{(l)}(\theta)=x\left((l-1) h^{\prime}+\theta\right), \quad 0 \leq \theta<h^{\prime}$. Then, $\mathcal{K}_{m}$ can be identified with $\left(\mathcal{K}_{m}^{\prime}\right)^{N}:=\mathcal{K}_{m}^{\prime} \oplus \cdots \oplus \mathcal{K}_{m}^{\prime}$, and we have $\|x\|_{\mathcal{K}_{m}}^{2}=\sum_{l=1}^{N}\left\|x^{(l)}\right\|_{\mathcal{K}_{m}^{\prime}}^{2}$. We denote the mapping from $x \in \mathcal{K}_{m}$ to $\check{x}:=\left(\left(x^{(1)}\right)^{T}, \cdots,\left(x^{(N)}\right)^{T}\right)^{T} \in\left(\mathcal{K}_{m}^{\prime}\right)^{N}$ by $\check{x}=\mathbf{L}_{N} x$, and call $\mathbf{L}_{N}$ fast-lifting.

Next, let us consider the mapping $\widetilde{\Gamma}_{N}: \mathcal{K}_{m_{1}} \rightarrow \mathcal{K}_{m_{2}}, x \mapsto y$ such that

$$
\left[\begin{array}{c}
y^{(1)}(\theta) \\
\vdots \\
y^{(N)}(\theta)
\end{array}\right]=\left[\begin{array}{ccc}
G_{11}(\theta) & \cdots & G_{1 N}(\theta) \\
\vdots & \ddots & \vdots \\
G_{N 1}(\theta) & \cdots & G_{N N}(\theta)
\end{array}\right]\left[\begin{array}{c}
x^{(1)}(\theta) \\
\vdots \\
x^{(N)}(\theta)
\end{array}\right], \quad 0 \leq \theta<h^{\prime}
$$

where $G_{k l}(\theta):\left[0, h^{\prime}\right) \rightarrow \mathbf{C}^{m_{2} \times m_{1}}, k, l=1, \cdots, N$ are measurable essentially bounded matrix functions. To be more precise, $\widetilde{\Gamma}_{N}$ is defined by

$$
\widetilde{\Gamma}_{N}=\mathbf{L}_{N}^{-1} G_{N} \mathbf{L}_{N}
$$

where $G_{N}:\left(\mathcal{K}_{m_{1}}^{\prime}\right)^{N} \rightarrow\left(\mathcal{K}_{m_{2}}^{\prime}\right)^{N}$ is given by (5). The class of operators given by (6) is denoted by $\widetilde{\boldsymbol{\Gamma}}_{N}$. We also define $\widetilde{\boldsymbol{\Gamma}}:=\cup_{N=1}^{\infty} \widetilde{\boldsymbol{\Gamma}}_{N}$.

Let us consider the special case with $G_{k l}(\theta)=0(k \neq l)$. Then, it is easy to see that $\widetilde{\Gamma}_{N}$ is nothing but the operator of multiplication by the matrix function $G(t)=G_{k k}\left(t-(k-1) h^{\prime}\right), \quad(k-1) h^{\prime} \leq t<k h^{\prime}$, which is obviously measurable and essentially bounded. Conversely, to any prescribed positive integer $N$ and to every operator of multiplication $\tilde{\Lambda}$ by a matrix function corresponds $G_{N}$ satisfying $G_{k l}(\theta)=0(k \neq l)$ such that $\widetilde{\Lambda}=\widetilde{\Gamma}_{N}$, where $\widetilde{\Gamma}_{N}$ is given by $(6)$. Thus, if we denote by $\tilde{\boldsymbol{\Lambda}}$ the class of operators of multiplication by a matrix function, then $\widetilde{\boldsymbol{\Gamma}}_{N} \supset \widetilde{\boldsymbol{\Lambda}}$ for any positive integer $N$. Note that the class $\boldsymbol{\Lambda}$ introduced in Definition 3 is a subset of $\widetilde{\boldsymbol{\Lambda}}$ with Hermitian matrix functions.

Now, let us introduce the class $\boldsymbol{\Gamma}_{N}$ defined as the subset of $\widetilde{\boldsymbol{\Gamma}}_{N}$ such that the associated matrix representation $G_{N}$ is Hermitian for each $\theta \in\left[0, h^{\prime}\right)$ :

$\boldsymbol{\Gamma}_{N}:=\left\{\mathbf{L}_{N}^{-1} G_{N} \mathbf{L}_{N}: G_{N}(\theta):=\left[G_{k l}(\theta)\right]_{k, l=1}^{N}\right.$ is measurable and essentially bounded, 


$$
\left.G_{N}(\theta)^{*}=G_{N}(\theta), \theta \in\left[0, h^{\prime}\right)\right\}
$$

Then, it is obvious that $\boldsymbol{\Gamma}_{N} \supset \boldsymbol{\Lambda}$ for any positive integer $N$. Finally, we define the significant class $\boldsymbol{\Gamma}:=\cup_{N=1}^{\infty} \boldsymbol{\Gamma}_{N}$, for which $\boldsymbol{\Gamma} \supset \boldsymbol{\Lambda}$ holds obviously.

The above inclusion property obviously implies that $\boldsymbol{\Gamma}$ can be regarded as a generalization of the class $\boldsymbol{\Lambda}$. This suggests a possibility that the class $\boldsymbol{\Lambda}$ in Definition 3 may be replaced by the new class $\boldsymbol{\Gamma}$, leading to an accordingly extended new class $\Theta\left(\supset \boldsymbol{\Theta}_{0}\right)$ given by

$$
\boldsymbol{\Theta}:=\{\Theta: \Theta=\Gamma+K, \Gamma \in \boldsymbol{\Gamma}, K \text { is linear self-adjoint and compact }\}(8)
$$

and that the statement of Theorem 4 could still be validated even if $\boldsymbol{\Theta}_{0}$ is replaced by $\boldsymbol{\Theta}$. This will indeed be justified in the following subsection. Before proceeding, we just state the following result, which is easy to prove.

Lemma 5 Every nonzero $\Gamma \in \boldsymbol{\Gamma}$ is a bounded noncompact operator.

We can thus ensure that every $\Theta \in \Theta$ has a unique representation $\Theta=\Gamma+K$ with $\Gamma \in \boldsymbol{\Gamma}$.

\subsection{General Robust Stability Theorem of Separator-Type}

We now give the following main result of this paper.

Theorem 6 Suppose that the assumptions in Theorem 4 are satisfied, and consider the class $\boldsymbol{\Theta}$ given by (8). Then, $\Sigma_{\Delta}$ is well-posed and internally stable for every $\Delta \in \boldsymbol{\Delta}$ if and only if there exists $\Theta \in \boldsymbol{\Theta}$ possibly dependent on $\zeta \in \partial \mathbf{D}$ and $\varepsilon>0$ possibly dependent on $\Delta$ satisfying (3) and (4).

Proof of Theorem 6. Consider $\Sigma_{0}$ with $P$ replaced by $P_{\Delta}$ in (2), and denote it by $\Sigma_{0 \Delta}$. Denote its transfer operator by $\widehat{G}_{\Delta}(\zeta):=\widehat{G}(\zeta) \widehat{\Delta}(\zeta)$, and apply the Nyquist stability criterion (Theorem 1) to study the stability of the closed-loop sampled-data system associated with $\Sigma_{0 \Delta}$ (which is nothing but $\left.\Sigma_{\Delta}\right)$. Recall that $\Theta \in \Theta$ has a unique representation $\Theta=\Gamma+K$ with $\Gamma \in \boldsymbol{\Gamma}$.

Sufficiency: Since a linear bounded self-adjoint operator is nonnegative definite if and only if its spectrum lies on the nonnegative real axis, and since the essential spectrum is a subset of the spectrum, it follows from (4) that the essential spectrum of

$$
T_{X}:=\left[\begin{array}{ll}
-D_{\Delta}^{*} & I
\end{array}\right] \Gamma\left[\begin{array}{c}
-D_{\Delta} \\
I
\end{array}\right]-\varepsilon I
$$

(or, that of $\mathbf{L}_{N} T_{X} \mathbf{L}_{N}^{-1}$ ) consists of only nonnegative numbers for any $\Delta \in \boldsymbol{\Delta}$, where $D_{\Delta}$ denotes the direct feedthrough matrix of $\Delta$. Here, $\mathbf{L}_{N} T_{X} \mathbf{L}_{N}^{-1}$ is an operator of multiplication by the matrix 


$$
X=\left[-I_{N} \otimes D_{\Delta}^{T} \quad I\right] G_{N}\left[\begin{array}{c}
-I_{N} \otimes D_{\Delta} \\
I
\end{array}\right]-\varepsilon I
$$

where $I_{N}$ denotes the $N \times N$ identity matrix and $\otimes$ denotes the Kronecker product. Hence, $T_{X}$ is isometrically isomorphic to the Laurent operator defined by the matrix (function) $X$, and thus it follows from Corollary XXIII.2.5 of Gohberg et al. (1990) that the spectrum of $T_{X}$ coincides with its essential spectrum, which consists of only nonnegative numbers, as mentioned above. Hence, $T_{X}$ is nonnegative definite. This implies that $X \geq 0$, and hence

$$
\left[-I_{N} \otimes D_{\Delta}^{T} I\right] G_{N}\left[\begin{array}{c}
-I_{N} \otimes D_{\Delta} \\
I
\end{array}\right]>0, \forall \Delta \in \boldsymbol{\Delta}
$$

Similarly, it follows from (3) that

$$
\left[\begin{array}{ll}
I & I_{N} \otimes D_{11}^{T}
\end{array}\right] G_{N}\left[\begin{array}{c}
I \\
I_{N} \otimes D_{11}
\end{array}\right] \leq 0
$$

Well-posedness of $\Sigma_{\Delta}$ for all $\Delta \in \boldsymbol{\Delta}$ now follows. To see this, suppose the contrary and assume that $\operatorname{det}\left(I+D_{11} D_{\Delta}\right)=0, \exists \Delta \in \boldsymbol{\Delta}$. Let us take $x \neq 0$ such that $\left(I+\left(I_{N} \otimes D_{11}\right)\left(I_{N} \otimes D_{\Delta}\right)\right) x=0$. Taking the quadratic forms with (11) and $x$, as well as (12) and $\left(I_{N} \otimes D_{\Delta}\right) x$, and then comparing the results, we are led immediately to contradiction because we have two values that coincide with each other but are positive and nonpositive at the same time.

Applying similar arguments to (3) and (4) leads immediately to that $I+$ $\widehat{G}_{\Delta}(\zeta)=I+\widehat{G}(\zeta) \widehat{\Delta}(\zeta)$ does not have an eigenvalue at 0 whenever $\Delta \in \boldsymbol{\Delta}$ and $\zeta \in \partial \mathbf{D}$. Since $I+\widehat{G}_{\Delta}(\zeta)=\left(I+D_{11} D_{\Delta}\right)\left(I+\widehat{G}_{\Delta}^{\prime}(\zeta)\right)$, it follows readily that $\widehat{G}_{\Delta}^{\prime}(\zeta)$ does not have an eigenvalue at -1 whenever $\Delta \in \boldsymbol{\Delta}$ and $\zeta \in \partial \mathbf{D}$, where $\widehat{G}_{\Delta}^{\prime}(\zeta)$ is defined in the same manner as the way $\widehat{G}^{\prime}(\zeta)$ is constructed from $\widehat{G}(\zeta)$. By the definition of the 2-regularized determinant, this implies that $\operatorname{det}_{2}\left(I+\widehat{G}_{\Delta}^{\prime}(\zeta)\right) \neq 0$ and thus the Nyquist locus of $\operatorname{det}_{2}\left(I+\widehat{G}_{\Delta}^{\prime}(\zeta)\right) \cdot \exp \left(\eta_{\Delta}(\zeta)\right)$, with $\eta_{\Delta}(\zeta)$ defined in the obvious fashion according to the definition of $\eta(\zeta)$, never goes through the origin whenever $\Delta \in \Delta$.

On the other hand, for $\Delta=0$, it follows that $\widehat{G}_{\Delta}^{\prime}(\zeta)=0$ and $\eta_{\Delta}(\zeta)=0$ so that $\operatorname{det}_{2}\left(I+\widehat{G}_{\Delta}^{\prime}(\zeta)\right) \cdot \exp \left(\eta_{\Delta}(\zeta)\right)=1$ regardless of $\zeta \in \partial \mathbf{D}$ (thus the Nyquist locus does not encircle the origin). The assumption (ii) on $\boldsymbol{\Delta}$ allows us to employ continuity arguments and thus this consequence together with the conclusion in the above paragraph leads to the claim that the Nyquist locus of $\Sigma_{0 \Delta}$ never encircles the origin whenever $\Delta \in \boldsymbol{\Delta}$. Since $\Sigma_{0 \Delta}$ is clearly internally stable by the assumptions, the assertion of this theorem follows readily from Theorem 1 .

Necessity: Suppose that $\Sigma_{\Delta}$ is well-posed and internally stable $\forall \Delta \in \boldsymbol{\Delta}$. Then, $\sigma\left(I+\widehat{G}_{\Delta}(\zeta)\right) \not \supset 0$, and thus $\sigma\left(I+\widehat{G}_{\Delta}(\zeta)^{*}\right) \not \supset 0$, whenever $\Delta \in \boldsymbol{\Delta}, \zeta \in \partial \mathbf{D}$ 
(recall Remark 2). Hence, $\sigma\left(\left(I+\widehat{G}_{\Delta}(\zeta)^{*}\right)\left(I+\widehat{G}_{\Delta}(\zeta)\right)\right) \not \supset 0, \forall \Delta \in \boldsymbol{\Delta}, \forall \zeta \in$ $\partial D$. Noting that (i) a linear bounded self-adjoint operator is nonnegative definite if and only if its spectrum lies on the nonnegative real axis, (ii) the spectrum of a linear bounded operator is compact, (iii) the spectrum of a linear self-adjoint operator is continuous under self-adjoint perturbations [Halmos (1982), p. 243], and (iv) $\partial \mathbf{D}$ is a compact set, there exists $\varepsilon>0$ dependent only on $\Delta$ such that $\left(I+\widehat{G}_{\Delta}(\zeta)^{*}\right)\left(I+\widehat{G}_{\Delta}(\zeta)\right) \geq \varepsilon I, \forall \zeta \in \partial \mathbf{D}$. Hence, taking

$$
\Theta(\zeta)=\left[\begin{array}{c}
-\widehat{G}(\zeta)^{*} \\
I
\end{array}\right][-\widehat{G}(\zeta) I]
$$

leads to (3) and (4). It is easily seen that this $\Theta$ belongs to $\boldsymbol{\Theta}$. Q.D.

Remark 7 Theorem 4 can also be proved similarly. It is not hard to see from the proof that the statement of Theorem 4 still remains valid as a necessary and sufficient condition even if the class $\boldsymbol{\Phi}$ in Definition 3 is restricted to consist of its real symmetric subset. Similarly, even if the matrix $G_{N}$ underlying the definition of the class $\boldsymbol{\Gamma}$ is restricted to be real, Theorem 6 remains valid. The assumption on $\Delta$ can easily be relaxed to allow h-periodic $\Delta$.

\section{Robust Stability Analysis with Noncausal LPTV Scaling}

This section illustrates the usefulness of Theorem 6. In particular, we demonstrate that it induces the novel idea that we call noncausal LPTV scaling, and show that it is quite effective for robust stability analysis of sampled-data systems.

For simplicity, we only consider the case $m=p$ (i.e., $D_{11}$ is square) and confine ourselves to the simplest case of noncausal LPTV scaling, which corresponds to the use of the separator $\Theta$ of the form

$$
\Theta=\left[\begin{array}{cc}
-\gamma^{2} \widetilde{\Gamma}_{N}^{*} \widetilde{\Gamma}_{N} & 0 \\
0 & \widetilde{\Gamma}_{N}^{*} \widetilde{\Gamma}_{N}
\end{array}\right], \widetilde{\Gamma}_{N} \in \widetilde{\boldsymbol{\Gamma}}_{N}, \widetilde{\Gamma}_{N}^{*} \widetilde{\Gamma}_{N}>0, \gamma>0
$$

for some positive integer $N$. The inequality (3) is then equivalent to $\left\|\tilde{\Gamma}_{N} \widehat{G}(z) \tilde{\Gamma}_{N}^{-1}\right\| \leq$ $\gamma(\forall \zeta \in \partial \mathbf{D})$. Similarly, (4) is equivalent to $\left\|\tilde{\Gamma}_{N} \widehat{\Delta}(z) \tilde{\Gamma}_{N}^{-1}\right\|<1 / \gamma(\forall \Delta \in$ $\boldsymbol{\Delta}, \forall \zeta \in \partial \mathbf{D})$ since $\varepsilon$ is allowed to depend on $\Delta$. Hence, the above separator corresponds to applying a "noncausally-scaled" (i.e., scaled with $\tilde{\Gamma}_{N}$ ) smallgain condition. Here, one might then argue that for the simplest noncausal LPTV scaling (14), such an argument is a direct consequence of the smallgain theorem and that Theorem 6 is not necessary to validate this sort of simplest noncausal LPTV scaling, but this is not the case. Indeed, the proof 
of the small-gain theorem is heavily dependent on causality of the systems composing the feedback loop (see, e.g., Vidyasagar (1993)). Note that the separator $\Theta$ in (14) in fact belongs to $\boldsymbol{\Gamma} \subset \boldsymbol{\Theta}$ (i.e., in the unique representation $\Theta=\Gamma+K$, the compact operator $K$ is zero).

It follows from the above consideration that if we assume the simple case $\Delta=\delta I$ for some scalar $\delta$, then (4) reduces to the condition $|\delta|<1 / \gamma$ while (3) reduces to the condition $\left\|\widetilde{\Gamma}_{N} \widehat{G}(\zeta) \widetilde{\Gamma}_{N}^{-1}\right\|_{\infty} \leq \gamma$, where $\|\cdot\|_{\infty}$ denotes the $H_{\infty}$ norm. Hence, we are led to the idea of computing $\gamma_{\min }=\inf \left\|\widetilde{\Gamma}_{N} \widehat{G}(\zeta) \widetilde{\Gamma}_{N}^{-1}\right\|_{\infty}$ where the infimum is taken over $\widetilde{\Gamma}_{N} \in \widetilde{\boldsymbol{\Gamma}}_{N}$. This is because stability of $\Sigma_{\Delta}$ is assured for all $|\delta|<1 / \gamma_{\min }$ then. As an example, let us consider the continuoustime (nominal) plant [Anderson and Moore (1990)] with transfer function

$$
\frac{1}{4 s^{2}} \cdot \frac{(s / a+1) \prod_{i=0}^{1}\left\{\left(s / \omega_{i}\right)^{2}+2 \zeta_{i}\left(s / \omega_{i}\right)+1\right\}}{\prod_{i=2}^{4}\left\{\left(s / \omega_{i}\right)^{2}+2 \zeta_{i}\left(s / \omega_{i}\right)+1\right\}}
$$

where $a=4.84, \zeta_{0}=0.02, \zeta_{1}=-0.4, \zeta_{2}=\zeta_{3}=\zeta_{4}=0.02, \omega_{0}=1, \omega_{1}=$ $5.65, \omega_{2}=0.765, \omega_{3}=1.41, \omega_{4}=1.85$, and the discrete-time stabilizing controller $\Psi$ obtained by applying the Tustin transformation at sampling period $h=3$ to the continuous-time controller $\Psi$ with transfer function

$$
-\frac{0.0513 s^{3}+0.00424 s^{2}+0.0296 s+0.00157}{s^{4}+0.693 s^{3}+0.779 s^{2}+0.293 s+0.0739}
$$

We then construct the generalized plant $P$ in such a way that its subsystem from $u$ to $y$ coincides with the nominal plant while the closed-loop mapping from $u$ to $y$ when $w$ is set to $-\delta z$ corresponds to the perturbed plant with $1 / a$ replaced by $(1+\delta) / a$, where $\delta$ is a real scalar. With this generalized plant $P$, we intend to analyze the allowable range of $\delta$ (and thus the parameter $a$ ) for which the sampled-data system remains internally stable. More precisely, we are interested in computing the largest $\delta_{\max }(>0)$ such that the closedloop sampled-data system $\Sigma_{\Delta}$ with $\Delta=\delta$ remains internally stable for all $\delta \in\left(-\delta_{\max }, \delta_{\max }\right)$.

A simple method for computing a lower bound of $\delta_{\max }$ is to apply the smallgain theorem. This corresponds to using the separator $\Theta=\operatorname{diag}\left[-\gamma^{2} I, I\right]$ and leads to a lower bound of $\delta_{\max }$ given by $\delta_{\mathrm{SG}}=11.0977$.

Another method for computing a lower bound of $\delta_{\max }$ is to apply the noncausal LPTV scaling suggested above. Assuming that the matrix representation $G_{N}(\theta)$ of $\widetilde{\Gamma}_{N}$ is constant with respect to $\theta$, the computation of the scaled $H_{\infty}$ norm $\left\|\widetilde{\Gamma}_{N} \widehat{G}(\zeta) \widetilde{\Gamma}_{N}^{-1}\right\|_{\infty}$ can be transformed into an equivalent discrete-time scaled $H_{\infty}$ norm computation in this example. This is because the subsystem from $w$ to $z$ is zero in this example, in which case it is well known that there exists an equivalent discretized generalized plant that is independent of " $\gamma$." More precisely, $\left\|\widetilde{\Gamma}_{N} \widehat{G}(\zeta) \widetilde{\Gamma}_{N}^{-1}\right\|_{\infty}$ is equal to the $H_{\infty}$ norm of the closedloop system consisting of the equivalent discretized generalized plant and the controller $\Psi$. Here, the scaling parameter can easily be extracted from the discretized generalized plant, and thus we are led to the discrete-time scaled 
Table 1

Lower bounds of $\delta_{\max }$ with LPTV scaling and computation times.

\begin{tabular}{c|cccc}
\hline$N$ & 2 & 3 & 5 & 10 \\
\hline$\delta_{\mathrm{LPTV}}^{\text {noncausal }}(N)$ & 17.3702 & 17.3784 & 17.3826 & 17.3846 \\
$\mathrm{CPU}$ time $(\mathrm{sec})$ & 16.18 & 23.19 & 45.47 & 237.11 \\
\hline$\delta_{\mathrm{LPTV}}^{\text {causal }}(N)$ & 11.4989 & 11.5168 & 11.5805 & 11.6508 \\
$\mathrm{CPU}$ time $(\mathrm{sec})$ & 7.77 & 10.23 & 18.71 & 77.86 \\
\hline
\end{tabular}

$H_{\infty}$-norm computation problem. Hence, by applying the well-known bounded real lemma, the minimization of $\left\|\widetilde{\Gamma}_{N} \widehat{G}(\zeta) \widetilde{\Gamma}_{N}^{-1}\right\|_{\infty}$ with respect to $\widetilde{\Gamma}_{N} \in \widetilde{\boldsymbol{\Gamma}}_{N}$ also reduces to a discrete-time problem, which in turn can be solved via LMI optimization in the matrix variable $G_{N}^{T} G_{N}$. The resulting lower bound for the case $\widetilde{\Gamma}_{N} \in \widetilde{\boldsymbol{\Gamma}}_{N}$ is denoted by $\delta_{\mathrm{LPTV}}^{\text {noncausal }}(N)$. The computation results of $\delta_{\mathrm{LPTV}}^{\text {noncausal }}(N)$ are shown in Table 1 (the upper rows), which clearly demonstrates the effectiveness of noncausal LPTV scaling compared with $\delta_{\mathrm{SG}}=11.0977$.

Remark 8 It would be worth noting that $\left\|\widetilde{\Gamma}_{N} \widehat{G}(\zeta) \widetilde{\Gamma}_{N}^{-1}\right\|_{\infty}$ has indeed been reduced by a suitable choice of $\widetilde{\Gamma}_{N}$, even though the unscaled system is SISO. This is due to the LPTV nature of the scaling we applied as well as that of the nominal open-loop sampled-data system $\Sigma_{0}$. We also remark here that the exact value of $\delta_{\max }$ is $\delta_{\max }=19.1842$ and thus the results in Table 1 are not contradictory in spite of noncausal operations in the analysis.

For reference, let us consider applying causal LPTV scaling [Hagiwara and Mori (2008)]. This corresponds to replacing $\widetilde{\Gamma}_{N}$ and $\widetilde{\boldsymbol{\Gamma}}_{N}$ with $\widetilde{\Lambda}_{N}$ and $\widetilde{\boldsymbol{\Lambda}}_{N}$ in (14), respectively. That is, we only consider LPTV but causal scaling of $\widehat{G}(\zeta)$. Except for this difference, we can repeat essentially the same procedure as in noncausal LPTV scaling to compute a lower bound of $\delta_{\max }$, which we denote by $\delta_{\mathrm{LPTV}}^{\text {causal }}(N)$. This corresponds to the analysis when Theorem 4 is available but Theorem 6 is not yet, and it is obvious that we have $\delta_{\mathrm{SG}} \leq$ $\delta_{\mathrm{LPTV}}^{\text {causal }}(N) \leq \delta_{\mathrm{LPTV}}^{\text {noncausal }}(N)$. Table 1 (the lower rows) shows the computation results of $\delta_{\mathrm{LPTV}}^{\text {causal }}(N)$. From this table, we can see that causal LPTV scaling also gives a less conservative result than the conventional small-gain analysis, but noncausal LPTV scaling is much more effective than causal LPTV scaling at a sacrifice of computational load (with Intel(R) Core(TM)2 Extreme CPU Q6800, 2.93GHz and 4GB memory).

\section{Conclusion}

In this paper, we derived a necessary and sufficient condition for robust stability of sampled-data systems, which is stated by using the notion of separators. The structure of the class $\boldsymbol{\Theta}$ of separators naturally led us to a novel idea of causal/noncausal LPTV scaling in robust stability analysis of sampled-data systems, and the effectiveness of such new types of scaling was demonstrated 
by a numerical example. We remark that further studies on the properties of causal/noncausal LPTV scaling in sampled-data systems have been conducted in Hagiwara and Mori (2008), Hagiwara (2006). Also, the associated numerical computation methods have been discussed in Hagiwara and Umeda (2007).

\section{References}

B. D. O. Anderson and J. B. Moore, 1990, Optimal Control: Linear Quadratic Methods, Prentice-Hall.

A. Böttcher and B. Silbermann, 1990, Analysis of Toeplitz Operators, SpringerVerlag.

T. Chen and B. Francis, 1995, Optimal Sampled-Data Control Systems, Springer.

I. Gohberg, S. Goldberg and M. A. Kaashoek, 1990, Classes of Linear Operators, Vol. I, Birkhäuser.

I. Gohberg, S. Goldberg and N. Krupnik, 2000, Traces and Determinants of Linear Operators, Birkhäuser.

T. Hagiwara, 2002, Nyquist stability criterion and positive-realness of sampled-data systems, Systems $\&$ Control Letters, Vol. 45, No. 4, pp. 283291.

T. Hagiwara, 2006, Causal/Noncausal Linear Periodically TimeVarying Scaling for Robust Stability Analysis and Their Properties, Proc. the 17th International Symposium on Mathematical Theory of Networks and Systems, pp. 742-752. http://www-ics.acs.i.kyoto-u.ac.jp/〜mtns2006/papers/0179.pdf

T. Hagiwara and R. Mori, 2008, Linear Periodically Time-Varying Scaling and Its Properties, Systems $\&$ Control Letters, Vol. 57, No. 10, pp. 816-821 (2008).

T. Hagiwara and Y. Tsuruguchi, 2004, Nyquist stability criterion of sampleddata systems with the 2-regularized determinants and its applications robust stability analysis, Proc. the 16th International Symposium on Mathematical Theory of Networks and Systems, THA3-4.

T. Hagiwara and H. Umeda, 2007, Robust stability analysis of sampled-data systems with noncausal periodically time-varying scaling: optimization of scaling via approximate discretization and error bound analysis, Prof. the 46th IEEE Conference on Decision and Control, pp. 450-457 (2007).

P. R. Halmos, 1982, A Hilbert Space Problem Book, Second Ed., Springer.

T. Iwasaki and S. Hara, 1998, Well-posedness of feedback systems: insights into exact robustness analysis and approximate computations, IEEE Transactions on Automatic Control, Vol. 43, No. 5, pp. 619-630.

M. Vidyasagar, 1993, Nonlinear Systems Analysis, Second ed., Prentice-Hall, NJ.

Y. Yamamoto, 1994, A function space approach to sampled-data systems and tracking problems, IEEE Trans. Automat. Contr., Vol. AC-39, No. 4, pp. 703-713.

J. Zhou and T. Hagiwara, 2005, 2-regularized Nyquist criterion in linear continuous-time periodic systems and its implementation, SIAM Journal on Control and Optimization, Vol. 44, No. 2, pp. 618-645. 